Pathologe 2008 · [Suppl 2] 29:255-258

DOI 10.1007/s00292-008-1097-9

Online publiziert: 10. Oktober 2008

(c) Springer Medizin Verlag 2008

H.P. Sinn · M. Andrulis · C. Mogler · P. Schirmacher Sektion für Gynäkologische Pathologie, Pathologisches Institut, Universitätsklinikum Heidelberg

\title{
Virtuelle Mikroskopie in Lehre und Ausbildung in der Pathologie
}

tion der Schnittpräparate Projektionsgeräte zur Darstellung von mikroskopischen Kurspräparaten auf. Zu erwähnen ist die Projektion der Schnittpräparate mit Geräten, die mit Hochvolt-Quecksilberdampflampen betrieben wurden, die in Heidelberg bis vor wenigen Jahren in Betrieb waren. In den 1970er und 1980er Jahren wurden an manchen Instituten geschlossene Diaprojektionskästen, die mit Tonbandkassetten gekoppelt waren, für das Selbststudium der Studenten eingesetzt. Solche und andere Technologien trugen zwar dazu bei, den Unterricht zu ergänzen, konnten sich jedoch aufgrund technischer Unzulänglichkeiten nicht behaupten und vor allem nicht die konventionelle Mikroskopie verdrängen. Erst durch die vollständige Digitalisierung histologischer Schnittpräparate in Mikroskopiescannern zusammen mit der Aufbereitung der Bilder in geeigneten digitalen Formaten und die Präsentation mit spezieller Software ist es möglich, eine der konventionellen Mikroskopie ebenbürtige Darstellung zu erzielen und diese in das Internet zu transportieren. Hier hat die VM das Potenzial, alles andere, einschließlich der Mikroskope zu verdrängen.

\section{Technische Aspekte}

Der Mikroskopscanner erzeugt Bilder für die VM mit hochauflösenden Aufnahmen, die in einer pyramidenartigen Dateistruktur mit multiplen Vergrößerungen gespeichert werden. Mit geeigneter Software können diese virtuellen Schnittpräparate in einer ähnlichen Weise wie mit einem realen Mikroskop betrachtet werden.
Während noch vor wenigen Jahren die Hard- und Software der VM vielfach auf Eigenentwicklungen basierte, gibt es nunmehr verschiedene kommerzielle Anbieter, die technisch ausgereifte Mikroskopscanner und darauf abgestimmte Software anbieten. Allerdings sind die angewandten Techniken von Hersteller zu Hersteller so unterschiedlich, dass weder die Dateiformate noch die WebAnwendungen untereinander kompatibel sind. Dadurch bedingt sind auch die Methoden und der Aufwand zur Einbindung der VM im Unterricht und im E-Learning sehr verschieden und reichen von sehr einfachen intuitiv bedienbaren Lösungen (hervorzuheben ist hier die Fa. Aperio) bis zu Anwendungen, die erhebliche technische Expertise und besondere Voraussetzungen an die EDV-technische Infrastruktur erfordern.

Die auf dem Markt befindlichen Scanner sind aufgrund der konventionellen Anforderungen an die Lichtmikroskopie für die Lehre allesamt prinzipiell als geeignet anzusehen und besitzen ähnliche Scangeschwindigkeiten. Unterschiede, die für die Darstellung der Bilder und für die Zukunftssicherheit relevant sind, betreffen folgende Punkte, auf welche bei der Auswahl eines Systems zu achten ist:

\section{Speicherung der Scans in einem Format, welches als kompatibel angesehen wird}

Dies trifft z. B. für das proprietäre „Composite-web-slide- (CWS-)Format" der Fa. Bacus zu, welches von mehreren Herstellern unterstützt wird; allerdings wird hier
VM verdrängt werden, ist ebenfalls lang. Im 20. Jahrhundert kamen für die Projek- 
jedes Bild in Tausende von Einzeldateien zerlegt, was die Verwaltung der VM zeitraubender macht als bei Aufnahmen, die in nur einer Datei abgelegt sind. Diese Dateiformate verbinden eine gängige Bildkompression, wie JPEG, mit einem offenen, nichtproprietären Dateiformat, wie TIFF. Zu nennen ist hier das SVS-Format der Fa. Aperio, in welchen JPEG -oder JPEG200o-komprimierte Bilder in einem speziellen TIFF-Format zusammengefasst werden. Für dieses weit verbreitete SVS-Format existieren Konversionsprogramme für die Umwandlung in andere Formate oder die Extraktion gewöhnlicher Einzelbilder, z. B. als JPEG. Durch die Verwendung eines kompatiblen oder eines offenen Bildformats kann sichergestellt werden, dass bei einem eventuellen Wechsel des Herstellers nicht die mit hohem Aufwand erzeugten Bildinformationen unbrauchbar werden.

\section{Konformität mit offenen Web-Standards}

Es zeigt sich, dass die Realisierung und Aktualisierung der E-Learning-Inhalte wesentlich davon abhängen, wie die Umsetzung der VM in Web-basierende Inhalte gelöst ist. Dies betrifft die Einbindung der VM in Web-basierende E-Learning-Systeme und den freien Web-Zugriff auf die VM. Für die Darstellung der VM im Intra- oder Internet gibt es verschiedene Lösungen, die entweder auf Java, Flash oder AJAX aufbauen, um das Einund Auszoomen und das Scrollen im Bild zu ermöglichen. Diese drei Methoden sind verbreitet, und der Student braucht daher in der Regel keine zusätzliche Software zu installieren, um die VM betrachten zu können. Allerdings unterscheiden sich die Java-, Flash- oder AJAX-basierenden Anwendungen in der Geschwindigkeit des Bildaufbaus und in der Kompatibilität mit Krankenhaus-Firewalls sowie in ihrer Erweiterbarkeit (Annotationen, „Screenshots“, Verlinkung mit anderen Inhalten u. a.). Die universellste Darstellungstechnik für die VM im WebBrowser basiert derzeit auf Adobe-Flash mit einem weit verbreiteten „Plug-in“ für Web-Browser.

\section{Praktische Umsetzung}

In Heidelberg führten wir die VM zunächst in den Kurs „Allgemeine Pathologie“ ein, mit dem Konzept eines kombinierten Unterrichts mit Beibehaltung der realen Mikroskopie im Kursraum und Hinzufügung der VM für die Projektion, für das Selbststudium und für die Nachbereitung der Inhalte. Diese Form der Ergänzung des klassischen Unterrichts wird auch als „blended learning“ bezeichnet. Für die Integration der VM in den Studentenunterricht verwenden wir zur effektiven Umsetzung und aus personellen Gründen ausschließlich die Standardsoftware des Herstellers des Mikroskopiescanners (Aperio, Cupertino/CA) und ein weit verbreitetes E-Learning-System (Moodle). Die Realisierung der ersten E-LearningKurse wurde in drei Phasen vollzogen (zeitlicher Aufwand in Klammern):

- Einscannen von Kurspräparaten bei 2ofacher Vergrößerung (1 Woche),

- Umsetzung der bestehenden Kursinhalte in ein E-Learning-System (3 Wochen),

- Aufbau einer Datenbank mit den virtuellen Kurspräparaten (virtueller Kurskasten), Erläuterungen und Bildannotationen (8 Wochen).

Das Einscannen bei 2ofacher Vergrößerung hat sich für fast alle Präparate als ausreichend erwiesen, wenige Objektträger wurden auch bei 4ofacher Vergrößerung gescannt. Die Auflösung ist bei 2ofacher Vergrößerung mehr als ausreichend, um die diagnostisch relevanten Details am Computermonitor darstellen zu können. Die Dateigröße der virtuellen Schnittpräparate beträgt durchschnittlich $500 \mathrm{MB}$ pro Bild (SVS-Format mit JPEGKompression), bei 4ofacher Vergrößerung über $1 \mathrm{~GB}$ pro Bild.

Aufgrund der strikten Firewall-Lösung des Universitätsklinikum Heidelberg wurden zwei Fileserver für die virtuellen Schnittpräparate benötigt: Ein interner Server, der sämtliche Bilddaten in einem RAID-5-Array speichert und nur innerhalb des Klinikum erreichbar ist. Dieser Server dient zum primären Abspeichern der Bildinformationen und für die Verwendung der VM im Kursraum. Die Bilder werden dann auf einen weiteren vom
Internet aus erreichbaren externer Server übertragen, auf den die Studenten von zu Hause aus zugreifen können. Auf Details zur Serverlösung wird in diesem $\mathrm{Zu}$ sammenhang nicht weiter eingegangen, es sei nur erwähnt, dass die Speicherung der Bilddaten und die Serversoftware betriebssystemunabhängig unter Windows (interner Server) bzw. Linux (externer Server) sind und dass zur Übertragung der Bilder eine spezielle Software (ImageServer von Aperio) erforderlich ist.

\section{Anwendungen im Studentenunterricht}

Ein fallbasierender, problemorientierter Pathologieunterricht wird seit Langem für effektiver gehalten als systematische Vorlesungen [1]. In solchen Seminaren, die eine kasuistische Darstellung mit der Systematik verbinden, oder auch in klassischen Kursen der allgemeinen oder speziellen Pathologie kann die VM mit Erfolg für die Darstellung der Histologie verwendet werden $[6,7]$. Dies betrifft den Einsatz der VM im Kursraum als Ersatz für die realen Mikroskope, die Projektion der Kurspräparate mit VM und die VM im Internet zur Rekapitulation der Lehrinhalte im Selbststudium. Auf diese Möglichkeiten und die jeweiligen Vor- und Nachteile soll im Folgenden eingegangen werden.

\section{Computerarbeitsplätze statt Kursmikroskope}

Der vollständige Ersatz von Kursmikroskopen durch Computerarbeitsplätze ist möglich, da die VM für Kurszwecke zumindest die gleichen Möglichkeiten bietet wie reale Mikroskope. Die Bedienung des PC fällt den Studenten erfahrungsgemäß leichter als die Mikroskopie, und die Einarbeitung in die Mikroskope und das Handling der Objektträger entfallen. Durch die gleichzeitige Darstellung der Übersicht über den gesamten Objektträger und einer Ausschnittsvergrößerung am Computer ist es für den Studenten leichter möglich, die Übersicht über ein Präparat $\mathrm{zu}$ behalten und $\mathrm{zu}$ navigieren. Alle Studenten haben die gleichen Schnittpräparate in optimaler Qualität zur Verfügung. Gegebenenfalls kann die 
Bilddarstellung an den Arbeitsplätzen mit der Projektion synchronisiert werden, sodass eine Präsentation durch den Tutor an den Arbeitsplätzen möglich ist. Im Übrigen eröffnen sich durch den Ersatz der Kursmikroskope durch PCs die Möglichkeiten der Verknüpfung der Mikroskopie mit vertiefenden Kursinhalten über eine geeignete E-Learning-Plattform und die Verwendung der PC-Arbeitsplätze für Selbstevaluation und Examina.

\section{Projektion der Kurspräparate}

Es bietet sich an, die VM auch für die Präsentation der Kurspräparate über einen Beamer zu verwenden. Dadurch entfällt die Notwendigkeit zur Videomikroskopie, und der technische Aufwand für die Projektion der Mikroskopie in den Kursen verringert sich insgesamt erheblich. Wird die VM auf ein interaktives Whiteboard projiziert, ergeben sich weitere Vorteile, wie bessere Präsenz des Lehrenden, und zusätzliche Möglichkeiten, wie Aufzeichnung der Darstellung, Hervorheben einzelner Inhalte der VM oder Annotationen über die Tafelfunktion des Whiteboards.

\section{E-Learning}

Am häufigsten wird die VM in der Lehre für die Bereitstellung der Präparate im Internet eingesetzt. Der Student erhält mit der VM die Möglichkeit der Vor- und Nacharbeitung der Kursinhalte von anderen Arbeitsplätzen in der Universität oder von zu Hause aus. Dies und die Verknüpfung der Inhalte mit theoretischen Erläuterungen in einem E-Learning-System kann können Lehrangebot erheblich verbreitern, ohne dass es zu einer zeitlichen Mehrbelastung für die Lehrenden kommt. Um der VM in diesem Kontext zum Erfolg zu verhelfen, ist es erforderlich, die $\mathrm{VM}$ in ein E-Learning-Angebot einzubetten, welches die VM inhaltlich begleitet und interpretiert. Die alleinige Darstellung der VM ohne Einblendung von Annotationen oder anderen Mikroskopierhilfen verwirrt erfahrungsgemäß viele Studenten, die das Mikroskopieren nicht gewöhnt sind und den „Wald vor lauter Bäumen" nicht erkennen. In diesem $\mathrm{Zu}$ sammenhang erscheint uns auch wichtig, die Bedeutung eines einfach navigier-

Pathologe 2008 · [Suppl 2] 29:255-258 DOI 10.1007/s00292-008-1097-9

(c) Springer Medizin Verlag 2008

\section{H.P. Sinn · M. Andrulis · C. Mogler · P. Schirmacher Virtuelle Mikroskopie in Lehre und Ausbildung in der Pathologie}

\section{Zusammenfassung}

Die Technik der virtuellen Mikroskopie (VM) erlaubt in gleicher Weise wie die konventionelle Mikroskopie das freie Begutachten histologischer Schnittpräparate am PC bei beliebiger Wahl der Bildausschnitte und der Vergrößerungen. Dies und die Möglichkeiten der Verknüpfung mit elektronischen Lehrinhalten und weiterführender Verlinkung macht die VM zum geeigneten Medium für Unterricht und Fortbildung in Pathologie in internetbasierenden Kurssystemen („E-Learning“). Anwendungen der VM in der Lehre betreffen kursbegleitendes Lernen („,blended learning") und Selbststudium im Internet sowie die Präsentation der VM im Kursraum bis hin zum Ersatz der Kursmikroskope durch PCs. Entscheidend für den Erfolg des Einsatzes der VM ist die didaktische Einbindung in entsprechend strukturierte virtuelle Kursräume und die interaktive Gestaltung der E-Learning-Inhalte. Weiterführende Anwendungen, die sich daraus ergeben bzw. darauf aufbauen, sind Lehrvideos bzw. Videoclips, "Podcasts", SCORM-Module und Präsentation der VM mit interaktiven „Whiteboards" im Kursraum.

\section{Schlüsselwörter}

Virtuelle Mikroskopie · Pathologieunterricht

\section{Virtual microscopy in pathology teaching and postgraduate training (continuing education)}

\section{Abstract}

As with conventional microscopy, virtual microscopy permits histological tissue sections to be viewed on a computer screen with a free choice of viewing areas and a wide range of magnifications. This, combined with the possibility of linking virtual microscopy to E-Learning courses, make virtual microscopy an ideal tool for teaching and postgraduate training in pathology. Uses of virtual microscopy in pathology teaching include blended learning with the presentation of digital teaching slides in the internet parallel to presentation in the histology lab, extending student access to histology slides beyond the lab. Other uses are student self-learning in the Internet, as well as the presentation of virtual slides in the classroom with or without replacing real microscopes. Successful integration of virtual microscopy depends on its embedding in the virtual classroom and the creation of interactive E-learning content. Applications derived from this include the use of virtual microscopy in video clips, podcasts, SCORM modules and the presentation of virtual microscopy using interactive whiteboards in the classroom.

\section{Keywords}

Virtual microscopy $\cdot$ Pathology teaching 
Tab. 1 Möglichkeiten für Referenzdatenbanken mit virtueller Mikroskopie. (Nach [4])

\begin{tabular}{|l}
\hline Studentenunterricht \\
Postgraduiertenunterricht \\
\hline Qualitätszirkel
\end{tabular}

Qualitätszirkel

Normale Histologie

Allgemeine und spezielle Histopathologie

Zytologie

Spezielle Themen der Organpathologie

Screening-Programme, z. B. Mamma
Immunhistochemie

baren, übersichtlichen Systems hervorzuheben, da bei einer zu starken Verschachtelung der Inhalte die möglichen Vorteile einer klaren Didaktik wieder zunichte gemacht werden können.

\section{Anwendungen im Postgraduier- tenunterricht, in Qualitätszirkeln und Referenzdatenbanken}

Beim Postgraduiertenunterricht liegt der Schwerpunkt auf fallbasierenden Darstellungen. Hier dient die VM in ähnlicher Weise wie beim Studentenunterricht der Vor- und Nachbearbeitung sowie der Fallpräsentation. Beispielhaft zu nennen sind hier die Internetseiten der USCAP („United States and Canadian Academy of Pathology“, http://www.uscap.org) und anderer internationaler Divisionen der IAP („International Academy of Pathology“). Während die Technik der Präsentation der VM hier gleich ist wie im Studentenunterricht, ist die Darstellung im Internet meist unterschiedlich (Einbettung in ELearning-Systeme beim Studentenunterricht und spezielle Webseiten beim Postgraduiertenunterricht). Vielfach wird gefragt, ob die technische Qualität der VM im Vergleich zur konventionellen Mikroskopie ausreichend ist für den Postgraduiertenunterricht. Vergleichende Untersuchungen haben jedoch gezeigt, dass für Pathologen die Beurteilung unbekannter histologischer und zytologischer Präparate in der VM gleich gut ist wie in der konventionellen Mikroskopie [2, 3]. Die Perspektive im Postgraduiertenunterricht ist der Aufbau großer virtueller Schnittsammlungen, die als Hintergrund zum Nachschlagen und für spezielle Fortbildungen dienen können. Ein Schritt ist diese Richtung ist das „Knowledge Hub for Pathology“ der USCAP [4], welches aber nicht systematisch gegliedert ist, sondern nach Seminaren.

Ähnliches gilt für Qualitätssicherungsmaßnahmen, wie Qualitätszirkel oder kli- nisch-pathologische Konferenzen. Die Konferenzen haben, ähnlich wie Fortbildungsmaßnahmen, zum Ziel, den Standard der histopathologischen Diagnostik zu verbessern und beruhen auf dem Versenden und/oder der Präsentation von Objektträgern. Aufgrund der damit verbundenen Probleme, wie der technische und logistische Aufwand, die Begrenzung der Teilnehmerzahl und die Unterschiede in Serienschnitten bietet sich auch hier die VM an. Zudem kann der konsequente Einsatz der VM für diese Zwecke zum Aufbau einer Referenzdatenbank seltenerer und histopathologisch gut dokumentierter Fälle genutzt werden. Die Verwendung der VM für Referenzzwecke ist viel sinnvoller als der Einsatz histopathologischer Bilddatenbanken, die es zahlreich im Internet gibt, da die Aussagekraft einzelner histologischer Aufnahmen immer begrenzt bleibt [5]. Weitere mögliche Anwendungen für Referenzdatenbanken sind in $\bullet$ Tab. 1 aufgeführt.

\section{Ausblick}

Die Möglichkeiten, die die VM eröffnet, können einen Qualitätssprung in der Lehre und in der Weiterbildung bewirken, wenn sie konsequent genutzt werden. Richtig eingesetzt können mit der VM nicht nur strukturelle Verbesserungen und eine verbesserte Transparenz der histopathologischen Diagnostik erreicht werden, sondern sie kann auch wesentlich zur Darstellung der Pathologie im Internet beitragen. Somit kann die VM als wichtiger Baustein zu einer Modernisierung der Methodik der Lehre in der Pathologie angesehen werden und $u$. a. dazu beitragen, den fachlichen Austausch zu verbessern und die gegenwärtigen Nachwuchsprobleme zu lösen.

\section{Korrespondenzadresse \\ Prof. Dr. H.P. Sinn \\ Sektion für Gynäkologische Pathologie \\ Pathologisches Institut \\ Universitätsklinikum Heidelberg \\ Im Neuenheimer Feld 220, 69120 Heidelberg \\ peter.sinn@med.uni-heidelberg.de}

Interessenkonflikt. Der korrespondierende Autor gibt an, dass kein Interessenkonflikt besteht.

\section{Literatur}

1. Bosman FT (1996) New curricula. J Pathol 180: 346-348

2. Dee FR, Donnelly A, Radio S et al. (2007) Utility of 2-D and 3-D virtual microscopy in cervical cytology education and testing. Acta Cytol 51:523-529

3. Dee FR, Lehman JM, Consoer D et al. (2003) Implementation of virtual microscope slides in the annual pathobiology of cancer workshop laboratory. Hum Pathol 34: 430-436

4. Gu J, Ogilvie RW (2005) Virtual microscopy and virtual slides in teaching, diagnosis, and research. CRC, Boca Raton

5. Jones KN, Kreisle R, Geiss RW et al. (2002) Group for research in pathology education online resources to facilitate pathology instruction. Arch Pathol Lab Med 126: 346-350

6. Kumar RK, Velan GM, Korell SO et al. (2004) Virtual microscopy for learning and assessment in pathology. J Pathol 204: 613-618

7. Marshall R, Cartwright N, Mattick K (2004) Teaching and learning pathology: a critical review of the English literature. Med Education 38: 302-313

8. Tuchman, AM (1993) Science, medicine and the state in Germany, the case of Baden, 1815-1871. Oxford University Press, New York 